\title{
THE STRICT DUAL OF $B^{*}$-ALGEBRAS
}

\author{
JOHN W. DAVENPORT
}

\begin{abstract}
If $A$ is a closed two-sided ideal in the $B^{*}$-algebra $X$, then $\left(X, \beta_{A}\right)^{*}$ with the strong topology is isomorphic to $A^{*}$, where $\beta_{A}$ is the strict topology on $X$.
\end{abstract}

Introduction. Let $C_{b}(X)$ be the Banach space of all real-valued bounded continuous functions on $X$. It is well known that if $X$ is compact then $\left(C_{b}(X), \sigma\right)^{*}$ is isomorphic to $M(X)$ where $M(X)$ is the bounded Radon measure on $X$ and $\sigma$ is the usual topology of uniform convergence. However, if $X$ is only locally compact Hausdorff then $\left(C_{b}(X), \sigma\right)^{*}$ is isomorphic to $M(\beta X)$ where $\beta X$ is the Stone-Čech compactification of $X$. In $1958, \mathrm{R}$. C. Buck [1] showed that if $C_{b}(X)$ is given the strict topology generated by $C_{0}(X)$, denoted as the $\beta$ topology, then $\left(C_{b}(X), \beta\right)^{*}$ is isomorphic to $M(X)$. We show that this result is essentially due to the fact that $C_{0}(X)$ is a closed two-sided ideal of $C_{b}(X)$.

1. Definition. Let $X$ be a $B^{*}$-algebra and $A$ a closed two-sided ideal in $X$. The strict topology of $X$ with respect to $A$, denoted as the $\beta_{A}$ topology on $X$, is the locally convex topology generated by the seminorms $\left(\lambda_{a}\right)_{a \in A}$ and $\left(\rho_{a}\right)_{a \in A}$ where $\lambda_{a}(x)=\|a x\|$ and $\rho_{a}(x)=\|x a\|$. We will denote $X$ with the $\beta_{A}$ topology by the pair $\left(X, \beta_{A}\right)$. It is clear that $\beta_{A}$ is a vector space topology in which multiplication is separately continuous.

2. Proposition. Let $A$ be a closed two-sided ideal in the $B^{*}$-algebra $X$. Then $A$ is $\beta_{A}$ dense in $X$.

Proof. C. E. Rickart [3] has shown that $A$ is an invariant subspace with respect to involution and, hence, $A$ can be considered as a $B^{*}$-algebra. If $\left\{e_{\alpha} \mid \alpha \in \Lambda\right\}$ is an approximate identity in $A\left[3\right.$, p. 245], then $e_{\lambda} x+x e_{\lambda}$ $e_{\lambda} x e_{\lambda}$ converges to $x$ in the $\beta_{A}$ topology for each $x \in X$. Since $A$ is a two-sided ideal, the set $\left\{e_{\lambda} x+x e_{\lambda}-e_{\lambda} x e_{\lambda} \mid \lambda \in \Lambda\right\}$ is contained in $A$ and, hence, the result follows.

3. Proposition. Let $A$ be a closed two-sided ideal in the $B^{*}$-algebra $X$. Then

$$
\left(X, \beta_{A}\right)^{*}=\left\{f \cdot a \mid a \in A, f \in X^{*}\right\} .
$$

Presented to the Society, October 18, 1974; received by the editors December 19,1975 and, in revised form, October $15,1976$.

AMS (MOS) subject classifications (1970). Primary 46B10, 46L05.

Key words and phrases. $B^{*}$-algebra, strict topology.

O American Mathematical Society 1977 
Proof. Let $f \in\left(X, \beta_{A}\right)^{*}$ and $\phi$ be the inclusion mapping of $A$ into $X$. Then $f_{\phi} \in A^{*}$. Since $A$ is a $B^{*}$-algebra there exists an $a \in A$ and $g \in A^{*}$ such that $f \phi=g \cdot a$ where $g \cdot a(x)=g(a x)$ [5]. By the Hahn-Banach theorem $g$ can be extended to an $h \in X^{*}$ such that $g=h \phi$. Let $\left\{e_{\alpha}\right\}$ be an approximate identity for $A$. Since $\left\{e_{\alpha} x+x e_{\alpha}-e_{\alpha} x e_{\alpha}\right\}$ converges to $x$ in the $\beta_{A}$ topology and $A$ is a closed two-sided ideal, we have that

$$
\begin{aligned}
f(x) & =\lim _{\alpha} f\left(e_{\alpha} x+x e_{\alpha}-e_{\alpha} x e_{\alpha}\right) \\
& =\lim _{\alpha} g \cdot a\left(e_{\alpha} x+x e_{\alpha}-e_{\alpha} x e_{\alpha}\right)=g(a x) \\
& =h(a x)=h \cdot a(x) .
\end{aligned}
$$

To get the reverse inclusion, it is sufficient to observe that if $x_{\alpha}$ converges to $x$ in the $\beta_{A}$ topology and $a \in A$, then $a x_{\alpha}$ converges to $a x$ in the norm topology. Thus, for $h \in X^{*}, h \cdot a\left(x_{\alpha}\right)$ converges to $h \cdot a(x)$ and, therefore, $h \cdot a$ is $\beta_{A}$ continuous.

4. Lemma. Let $A$ be a closed two-sided ideal in the $B^{*}$-algebra $X$ and $S$ a $\beta_{A}$ bounded subset of $X$. Then $\sup \{\|x a\|: x \in S, a \in A,\|a\|<1\}$ is finite.

Proof. For each $x \in S$, define $T_{x}$ from $A$ into $A$ by $T_{x} a=x a$. The map $T_{x}$ is clearly linear and continuous. Since $S$ is a $\beta_{A}$ bounded subset, for each $a \in A$, there exists an $M(a)>0$ such that $\sup _{x \in S}\left\{\left\|T_{x} a\right\|=\|x a\|\right\}<$ $M(a)$. Hence, by the uniform boundedness principle, there exists an $M>0$ such that $\left\|T_{x}\right\|<M$ for all $x \in S$. But

$$
\left\|T_{x}\right\|=\sup _{\|a\|<1}\left\|T_{x} a\right\|=\sup \{\|x a\|: a \in A,\|a\|<1\}
$$

and, hence, the Lemma follows.

The strong topology on $\left(X, \beta_{A}\right)^{*}$ is defined to be the topology of uniform convergence on the $\beta_{A}$ bounded subsets of $\left(X, \beta_{A}\right)$. Using this topology, we have the following result.

5. THEOREM. If $A$ is a closed two-sided ideal in the $B^{*}$-algebra $X$, then $\left(\left(X, \beta_{A}\right)^{*}, \gamma\right)$ is isomorphic to $A^{*}$, where $\gamma$ is the strong topology on $\left(X, \beta_{A}\right)^{*}$.

Proof. From Proposition 2 we have that $A$ is $\beta_{A}$ dense in $X$. Let $\phi$ be the inclusion mapping of $A$ into $X$ and let $\phi^{*}$ be the adjoint mapping of $\left(X, \beta_{A}\right)^{*}$ into $A^{*}$. We now show that $\phi^{*}$ is an isomorphism.

Since $A$ is $\beta_{A}$ dense in $X$, it follows that $\phi^{*}$ is one-to-one. Let $f \in A^{*}$. Then $f=g \cdot a$ for some $a \in A$ and $g \in A^{*}$ where $g \cdot a(x)=g(a x)$ [5]. By the Hahn-Banach theorem, we can extend $g$ to all of $X$ to obtain $\hat{g} \in X^{*}$ such that $\left.\hat{g}\right|_{A}=g$. Since $\phi^{*}(\hat{g} \cdot a)(b)=\hat{g} \cdot a(\phi(b))=g \cdot a(b)$ for all $b \in A$, it follows that $\phi^{*}$ is onto.

The continuity of $\phi^{*}$ is true since norm bounded sets are also $\beta$ bounded sets. To show that $\phi^{*-1}$ is continuous, it is sufficient to show that if $\left\{f_{n}\right\}$ is a sequence in $A^{*}$ that converges to $f \in A^{*}$, then $\phi^{*-1}\left(f_{n}\right)=F_{n}$ converges in the strong topology to $\phi^{*-1}(f)=F$. 
Let $S$ be a $\beta_{A}$ bounded set. By Lemma 4, there exists a number $M>0$ such that

$$
\sup \{\|x a\|: x \in S, a \in A \text { and }\|a\|<1\}<M .
$$

Let $\varepsilon>0$. Since $\left\{f_{n}\right\}$ converges to $f$ there exists an $N$ such that for all $n>N,\left\|f_{n}-f\right\|<\varepsilon / 3 M$. Since $\left\{f_{n} \mid n \in Z^{+}\right\} \cup\{f\}$ is totally bounded, for each $k>0$, there exists $a_{k} \in A, g_{k} \in A^{*}$ and $g_{n k} \in A^{*}$ satisfying

(5.1) $f_{n}=g_{n k} \cdot a_{k}$ and $f=g_{k} \cdot a_{k}$,

(5.2) $\left\|f_{n}-g_{n k}\right\|<1 / k$ and $\left\|f-g_{k}\right\|<1 / k$,

(5.3) $\left\|a_{k}\right\|<1$ [4].

Now $F_{n}=G_{n k} \cdot a_{k}$ and $F=G_{k} \cdot a_{k}$, where $G_{n k}$ is a Hahn-Banach extension of $g_{n k}$ and $G_{k}$ is a Hahn-Banach extension of $g_{k}$. We choose $k$ such that $1 / k<\varepsilon / 3 M$. The continuity of $\phi^{*-1}$ now follows from

$$
\begin{aligned}
\sup _{x \in S} \| & F_{n}(x)-F(x) \| \\
= & \sup _{x \in S}\left\|G_{n k} \cdot a_{k}(x)-G_{k} \cdot a_{k}(x)\right\|=\sup _{x \in S a_{k}}\left\|G_{n k}(x)-G_{k}(x)\right\| \\
< & \sup _{x \in S a_{k}}\left\|g_{n k}(x)-f_{n}(x)\right\|+\sup _{x \in S a_{k}}\left\|f_{n}(x)-f(x)\right\| \\
& \quad+\sup _{x \in S a_{k}}\left\|f(x)-g_{k}(x)\right\| \\
< & \sup _{\|x\|<M}\left\|g_{n k}(x)-f_{n}(x)\right\| \\
& \quad+\sup _{\|x\|<M}\left\|f_{n}(x)-f(x)\right\|+\sup _{\|x\|<M}\left\|f(x)-g_{k}(x)\right\| \\
< & \left\|g_{n k}-f_{n}\right\| M+\left\|f_{n}-f\right\| M+\left\|f-g_{k}\right\| M<\varepsilon .
\end{aligned}
$$

6. Corollary. Let $X$ be a locally compact space and $C_{b}(X)$ the algebra of bounded continuous real-valued functions on $X$. Let $A=C_{0}(X)$, the set of functions in $C_{b}(X)$ that vanish at infinity. Then $\left(C_{b}(X), \beta_{A}\right)^{*}$ is isomorphic to $M(X)$ where $M(X)$ is the set of bounded Radon measures on $X$.

Proof. This follows from Theorem 5 since $C_{0}(X)$ is a closed two-sided ideal in $C_{b}(X)$ and $C_{0}(X)^{*}=M(X)$ by the Riesz Representation Theorem.

This is R. C. Buck's result published in 1958 [1].

Let $A$ be a $B^{*}$-algebra. The double centralizer on $A$, denoted as $M_{d}(A)$, is the set of pairs $\left(T^{\prime}, T^{\prime \prime}\right)$ of mappings from $A$ into $A$ that satisfy $a\left(T^{\prime} b\right)=$ $\left(T^{\prime \prime} a\right) b$ for all $a, b \in A$. If $\left(T^{\prime}, T^{\prime \prime}\right) \in M_{d}(A)$, then $T^{\prime}$ and $T^{\prime \prime}$ are continuous linear maps on $A$ and $\left\|T^{\prime}\right\|=\left\|T^{\prime \prime}\right\|$, so that under the usual operations of addition and multiplication, $M_{d}(A)$ is a Banach algebra where $\left\|\left(T^{\prime}, T^{\prime \prime}\right)\right\|=$ $\left\|T^{\prime \prime}\right\|$. By defining $\left(T^{\prime}, T^{\prime \prime}\right)^{*}=\left(T^{\prime \prime *}, T^{\prime *}\right)$, where $T^{\prime *}(a)=\left(T^{\prime}\left(a^{*}\right)\right)^{*}$ and $T^{\prime \prime *}(a)=\left(T^{\prime \prime}\left(a^{*}\right)\right)^{*}$ for all $a \in A$, then $\left(T^{\prime}, T^{\prime \prime}\right)^{*} \in M_{d}(A)$ and thus $M_{d}(A)$ is a $B^{*}$-algebra.

If we define a map $\mu: A \rightarrow M_{d}(A)$ by the formula $\mu(a)=\left(L_{a}, R_{a}\right)$ where $L_{a}(x)=a x$ and $R_{a}(x)=x a$ for all $x \in A$, then $\mu$ is an isometric *- 
isomorphism from $A$ into $M_{d}(A)$ and $\mu(A)$ is a closed two-sided ideal in $M_{d}(A)$ [2]. If $A$ is commutative then $M_{d}(A)$ is isometrically *-isomorphic to the algebra of multipliers as studied by Wang [6]. For a more detailed account of the theory of double centralizers on a $B^{*}$-algebra, we refer the reader to [2].

7. Corollary. If $A$ is a $B^{*}$-algebra then $\left(M_{d}(A), \beta_{A}\right)^{*}$ under the strong topology is a Banach space that is isometrically isomorphic to $A^{*}$.

Proof. The isomorphism follows from Theorem 5, since $A$ can be considered as a closed two-sided ideal of $M_{d}(A)$. Since a $B^{*}$-algebra has an approximate identity uniformly bounded by one, it follows from Lemma 4 that the $\beta_{A}$ bounded subsets of $M_{d}(A)$ are norm bounded. Hence the strong topology on $\left(M_{d}(A), \beta_{A}\right)^{*}$ is the usual norm topology. The isometry follows from the fact that $f(X)=\lim _{\alpha} f\left(X \mu\left(e_{\alpha}\right)\right)$ where $f \in\left(M_{d}(A), \beta_{A}\right)^{*}, X \in$ $M_{d}(A)$ and $\left\{e_{\alpha}\right\}$ is an approximate identity on $A$.

This is D. C. Taylor's result published in 1970 [5].

\section{REFERENCES}

1. R. C. Buck, Bounded continuous functions on a locally compact space, Michigan Math. J. 5 (1958), 95-104. MR 21 \# 4350.

2. R. C. Busby, Double centralizers and extensions of $C^{*}$-algebras, Trans. Amer. Math. Soc. 132 (1968), 79-99. MR 37 \#770.

3. C. E. Rickart, General theory of Banach algebras, Van Nostrand, Princeton, N.J., 1960. MR 22 \# 5903.

4. D. C. Taylor, A characterization of Banach algebras with approximate unit, Bull. Amer. Math. Soc. 74 (1968), 761-766. MR 37 \#2003.

5. The strict topology for double centralizer algebras, Trans. Amer. Math. Soc. 150 (1970), 633-643. MR 44 \# 7302.

6. J.-K. Wang, Multipliers of commutative Banach algebras, Pacific J. Math. 11 (1961), 1131-1149. MR 25 \# 1462.

Department of Mathematics, James Madison University, Harrisonburg, Virginia 22801 